\section{Erythrocyte structure and dynamics quantified by Hilbert phase microscopy}

\author{
Gabriel Popescu* \\ Massachusetts Institute of Technology \\ George R. Harrison Spectroscopy Laboratory \\ Cambridge, Massachusetts 02139
}

\section{Takahiro Ikeda}

Hamamatsu Photonics K.K. 5000 Hirakuchi, Hamamatsu

Shizuoka 434-8601 Japan

\section{Catherine A. Best}

Harvard Medical School and Massachusetts General Hospital

Department of Pathology

Boston, Massachusetts 02114

\section{Kamran Badizadegan}

Harvard Medical School and Massachusetts General Hospital

Boston, Massachusetts 02114

\author{
Ramachandra R. Dasari \\ Michael S. Feld \\ Massachusetts Institute of Technology \\ George R. Harrison Spectroscopy Laboratory \\ Cambridge, Massachusetts 02139
}

\begin{abstract}
We present a new quantitative method for investigating red blood cell morphology and dynamics. The instrument integrates quantitative phase microscopy with an inverted microscope, which makes it particularly suitable for the noninvasive assessment of live erythrocytes. In particular, we demonstrate the ability of this approach to quantify noninvasively cell volume and dynamic morphology. The subnanometer path-length sensitivity at the millisecond time scales is exemplified by measuring the hemoglobin flow out of the cell during hemolysis. ๑) 2005 Society of Photo-Optical Instrumentation Engineers. [DOI: 10.1117/1.2149847]
\end{abstract}

Keywords: microscopy; cells; phase retrieval; medical imaging.

Paper 05252LRR received Aug. 26, 2005; revised manuscript received Nov. 1, 2005; accepted for publication Nov. 3, 2005; published online Dec. 30, 2005.

Various biological phenomena, such as red blood cell (RBC) membrane fluctuations, develop at the nanometer and millisecond scales and their quantitative investigation is extremely challenging. An example of these phenomena is represented by the RBC thermal fluctuations, which have been first observed many years ago. ${ }^{1}$ These motions are typically of

*Tel: 617-258-7831; E-mail: gpopescu@mit.edu

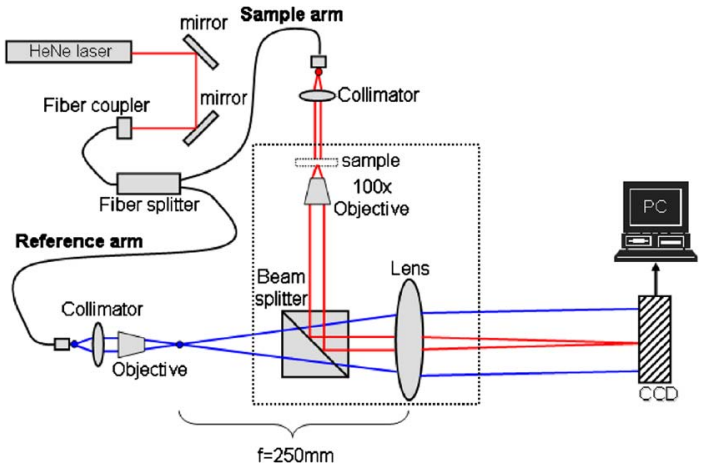

Fig. 1 Experimental setup.

the order of tens of nanometers over a broad range of frequencies. Significant progress has been made over the past years on modeling this phenomenon, in an effort to connect the relevant mechanical coefficients of the cell to the statistical properties of the fluctuations. ${ }^{1,2}$ However, the nature of these fluctuations is not completely elucidated. It has been suggested that thermal excitation is not entirely responsible for these motions and that a deterministic component may in fact accomplish certain physiological tasks, such as improving the cell flexibility and filterability. ${ }^{1,3}$ Development of quantitative and nonperturbative techniques for studying such phenomena is highly needed.

Phase contrast microscopy ${ }^{1}$ and reflection interference contrast microscopy ${ }^{4}$ have been used previously to measure dynamic changes in the shape of RBCs. However, these methods are not inherently quantitative. Thus, 3-D quantitative erythrocyte shape measurements have been limited to atomic force and scanning electron microscopy. Because of the limitations imposed by the sample preparation, these techniques have limited applicability to cells under physiological and dynamic conditions. ${ }^{5}$ Fourier phase microscopy (FPM) has been developed in our laboratory as a modality to extract quantitative phase images with subnanometer path-length sensitivity over time periods from seconds to a cell life cycle; ${ }^{6}$ its potential for studying the structure and low-frequency fluctuations of RBCs has been demonstrated.

In this letter, we present a new quantitative phase microscope dedicated to the quantitative assessment of RBC morphology and dynamics. The instrument integrates a typical inverted microscope with the principle of Hilbert phase microscopy (HPM). HPM extends the concept of complex analytic signals to the spatial domain and measures quantitative phase images from only one spatial interferogram recording, as demonstrated recently on test samples. ${ }^{7}$ Due to its singleshot nature, the HPM acquisition time is limited only by the recording device and thus can be used to accurately quantify nanometer-level path-length shifts at the millisecond time scales or less, where many relevant biological phenomena develop.

The experimental setup is shown in Fig. 1. A HeNe laser $(\lambda=633 \mathrm{~nm})$ is coupled into a $1 \times 2$ single-mode fiber optic coupler and collimated on each of the two outputs. One output field acts as the illumination field for an inverted microscope equipped with a $100 \times$ objective. All the optical fibers are

1083-3668/2005/10(6)/060503/3/\$22.00 @ 2005 SPIE 

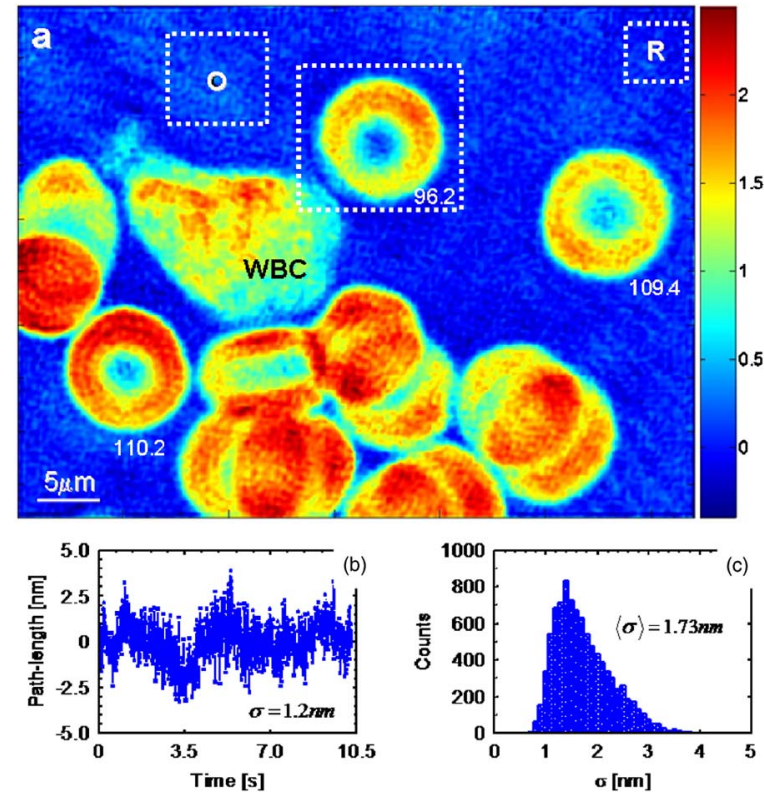

Fig. 2 (a) Quantitative phase image of whole blood smear; the volumes of RBCs are indicated in femtoliters and the color bar is in radians. (b) Path-length fluctuations of an arbitrary point within area O. The standard deviation $\sigma$ is indicated. (c) Histogram of standard deviations corresponding to all the points within region $\mathrm{O}$. The average standard deviation $\langle\sigma\rangle$ is indicated.

fixed to minimize phase noise. The tube lens is such that the image of the sample is formed at the CCD plane via the beamsplitter cube. The second fiber coupler output is collimated and expanded by a telescopic system consisting of another microscope objective and the tube lens. This reference beam can be approximated by a plane wave, which interferes with the image field. The reference field is tilted with respect to the sample field such that uniform fringes are created at an angle of $45 \mathrm{deg}$ with respect to $x$ and $y$ axes. The CCD used (C7770, Hamamatsu Photonics) has an acquisition rate of $291 \mathrm{frames} / \mathrm{s}$ at the full resolution of $640 \times 480$ pixels, at $1-1.5 \mathrm{~ms}$ exposure time. The fringes are sampled by 6 pixels per period. The spatial irradiance associated with the interferogram across one direction is given by

$$
I(x)=I_{R}+I_{S}(x)+2 \sqrt{I_{R} I_{S}(x)} \cos [q x+\phi(x)],
$$

where $I_{R}$ and $I_{S}$ are, respectively, the reference and sample irradiance distributions, $q$ is the spatial frequency of the fringes, and $\phi$ is the spatially varying phase associated with the object, the quantity of interest in our experiments. Using high-pass spatial filtering and Hilbert transformation, the quantity $\phi$ is retrieved in each point of the single-exposure image.

To exemplify the ability of the new instrument to perform RBC dynamic morphometry at the millisecond and nanometer scales, we obtained time-resolved HPM images of RBCs. Droplets of whole blood were simply sandwiched between cover slips, with no additional preparation. Figure 2 shows a quantitative phase image of live blood cells; both isolated and agglomerated erythrocytes are easily identifiable. A white blood cell (WBC) is also present in the field of view. Using the refractive index of the cell and surrounding plasma of 1.40
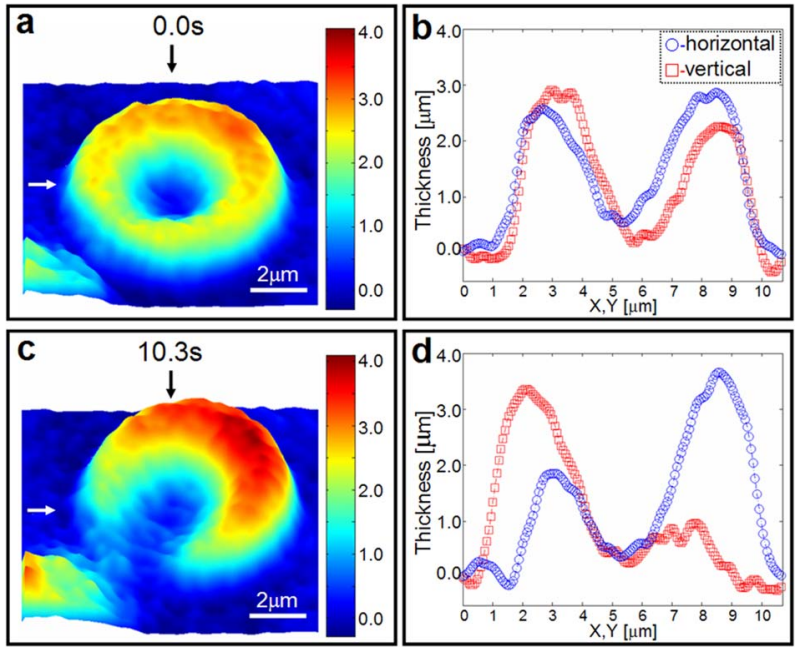

Fig. 3 Quantitative assessment of the shape transformation associate with the RBC selected in Fig. 2, during a 10-s period. The profiles in (b) and (d) are measured along the profiles indicated by the arrows in (a) and (c).

and 1.34 , respectively, ${ }^{8}$ the phase information associated with the RBCs can be easily translated into nanometer scale image of the cell topography. The assumption of optical homogeneity of RBC is commonly used ${ }^{1,4}$ and justified by the knowledge that cellular content consists mainly of hemoglobin solution. The dynamic thickness profile of the cell can be directly obtained from the phase measurement, $u(x, y ; t)$ $=(\lambda / 2 \pi \Delta n) \phi(x, y ; t)$, where $\Delta n$ is the refractive index contrast between the hemoglobin contained in the cell and the surrounding fluid. Thus the instantaneous volume of individual cells can be measured from the HPM data, $V(t)$ $=\int u(x, y ; t) \mathrm{d} x \mathrm{~d} y$. During our rapid measurements, errors in the volume measurement due to changes in refractive index are not likely to be significant. Spatial inhomogeneities of the imaging fields may affect the accuracy of the volume measurement and this influence was minimized by accurately spatially filtering and collimating the beams. In Fig. 2, the measured volumes (units of in femtoliters) are displayed below individual RBCs.

In order to eliminate the longitudinal noise between successive frames, each phase image was referenced to the average value across an area in the field of view containing no cells [denoted in Fig. 2(a) by R]. To quantify the residual noise of the instrument in a spatially relevant way, we recorded sets of 1000 images, acquired at $10.3 \mathrm{~ms}$ each and analyzed the path-length fluctuations of individual points within an $100 \times 100$ pixel area [denoted in Fig. 2(a) by O]. The path length associated with each point in $\mathrm{O}$ was averaged over $5 \times 5$ pixels, which approximately corresponds to the dimensions of the diffraction limit spot. Figure 2(b) shows the temporal path-length fluctuations of an arbitrary point within the region $\mathrm{O}$, while the histogram of the standard deviations associated with all the spots within region $\mathrm{O}$ is shown in Fig. 2(c). The average value of this histogram is indicated. This noise assessment demonstrates that our HPM instrument is capable of providing quantitative information about structure and dynamics of biological systems, such as RBCs, at the nanometer scale. 


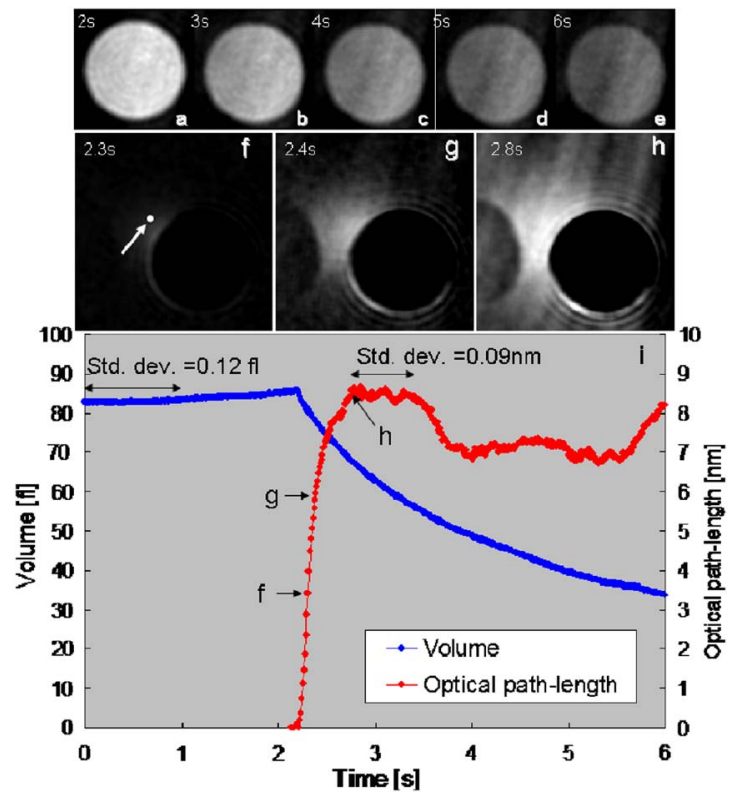

Fig. 4 (a)-(e). Various stages of hemolysis during a 6-s period. (f)-(h) Phase image of hemoglobin expelled from the cell corresponding to various points in time, as indicated. (i) Cell volume change and optical path-length shift associated with a point outside the cell (indicated by the arrow) during the 6-s period.

An example of significant dynamical change of a live RBC is shown in Figs. 3(a) and 3(c). The phase images correspond to the RBC shown in Fig. 2(a) and are acquired $10.3 \mathrm{~ms}$ apart and represent the first and the last frames in a 1,000 frame data set. Figures 3(b) and 3(d) show the horizontal and vertical thickness profiles of the cell at the two stages, with nanometer accuracy. Interestingly, the significant change in the cell shape is due to a rapid interaction with the neighboring WBC, at the lower left corner of the image [also shown as WBC in Fig. 2(a)]. This results in a rapid asymmetric shape change that is easily quantified by HPM. We note that such dynamic modifications in cells under physiological conditions cannot be quantified by techniques such as atomic force or electron microscopy.

Hemolysis (RBC "lysing") is a spectacular phenomenon in which the erythrocyte membrane ruptures and the cell looses its hemoglobin content. This process has been studied recently in the context of optical clearing. ${ }^{9}$ Using the HPM instrument, we employed a sequence of 1,000 phase images, at $10.3 \mathrm{~ms}$ acquisition time, to dynamically quantify the changes in the cell as a result of lysing. Figures 4(a)-4(e) depict the cell volume decrease during various stages of hemolysis. Note the abnormal flat shape of the cell. The phase shifts due to the expelled hemoglobin can be observed in Figs. 4(f)-4(h), where only the region surrounding the cell is represented, to avoid gray-scale saturation. The membrane rupture is highly localized, as indicated by the asymmetry in Figs. 4(f)-4(h), and the hemoglobin appears to be diffusing from a point source on the cell. The RBC volume was evaluated during the process and its temporal dependence is plotted in Fig. 4(i). It appears that during this highly dynamic process, the volume of the cell decreases by $50 \%$ in less than $4 \mathrm{~s}$. Before the onset of hemolysis, the standard deviation of the volume measurement has a value of $0.12 \mathrm{fl}$, as indicated. On the other hand, the path-length shift associated with a point in close proximity to the cell reaches a steady-state maximum level of almost $9 \mathrm{~nm}$ in about $1 \mathrm{~s}$. In order to improve the signal to noise of this ultrasensitive measurement, the signal was averaged in space over $11 \times 11$ pixels and in time over 10 frames. The resulting standard deviation of these data reached the remarkably low value of $0.09 \mathrm{~nm}$. The origin of the slight oscillation present in this curve is not well understood.

The instrument presented here requires significantly less computational work than digital holography, which relies on numerical Fresnel field propagation. ${ }^{10,11}$ In addition, as a single-shot technique, it provides faster images than reported with other quantitative phase imaging techniques. ${ }^{12,13}$ It can be suitably adjusted to allow for automatic RBC measurements of shape and volume, which in turn have diagnostic relevance.

\section{Acknowledgments}

This work was carried out at the MIT Laser Biomedical Research Center, supported by NIH P41 RR 02594 and partially by Hamamatsu Photonics K.K.

\section{References}

1. F. Brochard and J. F. Lennon, "Frequency spectrum of the flicker phenomenon in erythrocytes," J. Phys. (Paris) 36, 1035-1047 (1975).

2. N. Gov, "Membrane undulations driven by force fluctuations of active proteins," Phys. Rev. Lett. 93(26), 268104 (2004); R. Lipowski and M. Girardet, "Shape fluctuations of polymerized or solidlike membranes," Phys. Rev. Lett. 65, 2893-2896 (1990).

3. S. Tuvia, S. Levin, and R. Korenstein, "Correlation between local cell-membrane displacements and filterability of human red-bloodcells," FEBS Lett. 304(1), 32-36 (1992).

4. A. Zilker, H. Engelhardt, and E. Sackmann, "Dynamic reflection interference contrast (RIC-) microscopy-a new method to study surface excitations of cells and to measure membrane bending elasticmoduli," J. Phys. (Paris) 48(12), 2139-2151 (1987).

5. R. Nowakowski, P. Luckham, and P. Winlove, "Imaging erythrocytes under physiological conditions by atomic force microscopy," Biochim. Biophys. Acta 1514(2), 170-176 (2001); P. Matarrese, E. Straface, D. Pietraforte, et al., "Peroxynitrite induces senescence and apoptosis of red blood cells through the activation of aspartyl and cysteinyl proteases," FASEB J. 19(3), 416-418 (2005).

6. G. Popescu, L. P. Deflores, J. C. Vaughan, et al., "Fourier phase microscopy for investigation of biological structures and dynamics," Opt. Lett. 29(21), 2503-2505 (2004).

7. T. Ikeda, G. Popescu, R. R. Dasari, et al., "Hilbert phase microscopy for investigating fast dynamics in transparent systems," Opt. Lett. 30(10), 1165-1168 (2005).

8. M. Hammer, D. Schweitzer, B. Michel, et al., "Single scattering by red blood cells," Appl. Opt. 37(31), 7410-7418 (1998).

9. V. V. Tuchin, D. M. Zhestkov, A. N. Bashkatov, et al., "Theoretical study of immersion optical clearing of blood in vessels at local hemolysis," 12(13), 2966-2971 (2004).

10. D. Carl, B. Kemper, G. Wernicke, et al., "Parameter-optimized digital holographic microscope for high-resolution living-cell analysis," Appl. Opt. 43(36), 6536-6544 (2004).

11. P. Marquet, B. Rappaz, P. J. Magistretti, et al., "Digital holographic microscopy: a noninvasive contrast imaging technique allowing quantitative visualization of living cells with subwavelength axial accuracy," Opt. Lett. 30(5), 468-470 (2005).

12. G. A. Dunn and D. Zicha, in Cell Biology: A Laboratory Handbook, J. Celis, Ed., Academic Press, New York, (1997).

13. D. Paganin and K. A. Nugent, "Noninterferometric phase imaging with partially coherent light," Phys. Rev. Lett. 80(12), 2586-2589 (1998). 